\title{
Effects of dietary fiber levels on cecal microbiota composition in geese
}

\author{
Yanpin $\mathrm{Li}^{1,2}$, Haiming Yang ${ }^{1}$, Lei $\mathrm{Xu}^{1}$, Zhiyue Wang ${ }^{1, *}$, Yue Zhao ${ }^{1}$, and Xiaoshuai Chen ${ }^{1}$
}

* Corresponding Author: Zhiyue Wang Tel: +86-514-87979045, Fax: +86-514-87990256,

E-mail: dkwzy@263.net

1 Department of Animal Science and Technology, Yangzhou University, Yangzhou, Jiangsu 225009, China

${ }^{2}$ Feed Research Institute, Chinese Academy of Agricultural Sciences, Beijing 100081, China

\section{ORCID}

Yanpin Li

https://orcid.org/0000-0002-2522-0566 Haiming Yang

https://orcid.org/0000-0003-2901-6113 Lei Xu

https://orcid.org/0000-0002-9676-3971

Zhiyue Wang

https://orcid.org/0000-0002-2532-7363

Yue Zhao

https://orcid.org/0000-0002-0924-4859

Xiaoshuai Chen

https://orcid.org/0000-0001-5836-4673

Submitted Dec 18, 2017; Revised Jan 13, 2018; Accepted Jan 26, 2018
Objective: This study shows the effects of dietary fiber levels on cecal microbiota composition in geese at day 70 according to pyrosequencing of the $16 \mathrm{~S}$ ribosomal RNA gene.

Methods: A total of 468 1-day-old healthy male Yangzhou goslings with similar body weight were randomly divided into 3 groups with 6 replicates per group and 26 geese per replicate. Geese were fed diets with fiber levels of 2.5\% (low fiber level diet, Group I) and 6.1\% (Group III) during days 1-70, respectively, or 4.3\% for days 1-28 and 6.1\% for days 29-70 (Group II). Results: Low fiber level diet decreased body weight, average daily gain during, increased lower feed conversation rate of geese during day 1 to $70(p<0.05)$. Low fiber level diet decreased the total operational taxonomic units, Chaol index and Shannon index, whereas increased the Simpson index of cecal microbiota in geese at day 70. Low fiber level diet decreased the relative abundance of Bacteroidetes, Firmicutes, Bacteroides, and Paraprevotella in cecum of geese at day 70. The similarity of cecal microbiota between low fiber level diet group and other groups was smaller.

Conclusion: This study indicates that the low fiber level diet decreased diversity of microbiota, and relative abundance of some beneficial microbiota in cecum of geese at day 70, implying that the low fiber level diet has negative influence on performance by altering the diversity and population of cecal microbiota in geese.

Keywords: Fiber; Goose; Cecum; Microbiota

\section{INTRODUCTION}

Unlike other avian species, the goose is a kind of waterfowl with a relatively developed paired cecum, which can partly take advantage of fibrous plant materials [1]. And birds can digest fiber only through fermentation, mainly in the cecum [2]. The cecum of geese plays a very important role in nutritional digestion when the geese are fed with low energy and high fiber diets, as reported by Garcia et al [3]. In addition, the utilization of acid detergent fiber and neutral detergent fiber were significantly decreased after the cecum was removed from geese fed the basal diet in the study of Yang et al [4]. However, poultry cannot secrete cellulose decomposition enzyme or semi cellulose decomposition enzyme, those enzymes are mainly secreted by microbiota in the digestive tract. Thus it is particularly important to study the response of cecal microbiota to dietary fiber.

Crude fiber is the main component of plant cell walls, and it has double effects on animal nutrition. When the fiber level is in the appropriate range, it can maintain the normal structure and function of intestinal tract, prevent pica and improve meat quality. Too high or too low fiber level will affect the digestion and absorption of nutrients. However, the mechanism of dietary fiber effect on the production performance in geese is not clear. Previous studies [5,6] mainly studied the effects of dietary fiber on production performance, intestinal 
morphology, gastrointestinal-tract development of geese, in order to select the appropriate levels and sources of fiber in geese. Nevertheless, only a few studies were carried out to investigate the influence of dietary fiber on cecal microbiota composition in geese.

Before the present experiment, we have investigated the effects of dietary fiber on growth performance, slaughter performance, serum biochemical parameters, and nutrient utilization in geese [7]. But the mechanism of dietary fiber affecting the performance needs further research. We speculate that dietary fiber affects the performance of geese through the cecal microbiota. Thus the present study was carried out to investigate the effects of dietary fiber levels on cecal microbiota composition in geese, in order to provide a theoretical basis for the mechanism of the fiber digestion of geese.

\section{MATERIALS AND METHODS}

\section{Experimental design and diets}

Table 1. Ingredients and nutrient composition of experimental diets for days 1 to 28 (as fed)

\begin{tabular}{|c|c|c|c|}
\hline Item & Group I) & Group II & Group III) \\
\hline \multicolumn{4}{|l|}{ Ingredients (\%) } \\
\hline Corn & 63.20 & 63.20 & 63.20 \\
\hline Soybean meal & 25.00 & 25.00 & 25.00 \\
\hline Corn protein meal & 3.20 & 3.20 & 3.20 \\
\hline Cellulose & - & 1.86 & 3.72 \\
\hline Limestone & 1.00 & 1.00 & 1.00 \\
\hline Calcium hydrogen phosphate & 1.00 & 1.00 & 1.00 \\
\hline Methionine & 0.10 & 0.10 & 0.10 \\
\hline Salt & 0.30 & 0.30 & 0.30 \\
\hline Vermiculite & 5.20 & 3.34 & 1.48 \\
\hline Vitamin and trace mineral premix $x^{2)}$ & 1.00 & 1.00 & 1.00 \\
\hline \multicolumn{4}{|l|}{ Nutrient composition ${ }^{3)}$} \\
\hline Metabolizable energy (MJ/kg) & 11.54 & 11.54 & 11.54 \\
\hline Crude protein (\%) & 18.56 & 18.56 & 18.56 \\
\hline Crude fiber (\%) & 2.52 & 4.32 & 6.13 \\
\hline Neutral detergent fiber (\%) & 8.67 & 10.41 & 12.20 \\
\hline Acid detergent fiber (\%) & 4.32 & 6.09 & 7.62 \\
\hline Calcium (\%) & 0.75 & 0.75 & 0.75 \\
\hline Total phosphorus (\%) & 0.57 & 0.57 & 0.57 \\
\hline Lysine (\%) & 0.85 & 0.85 & 0.85 \\
\hline Methionine (\%) & 0.39 & 0.39 & 0.39 \\
\hline \multicolumn{4}{|c|}{ 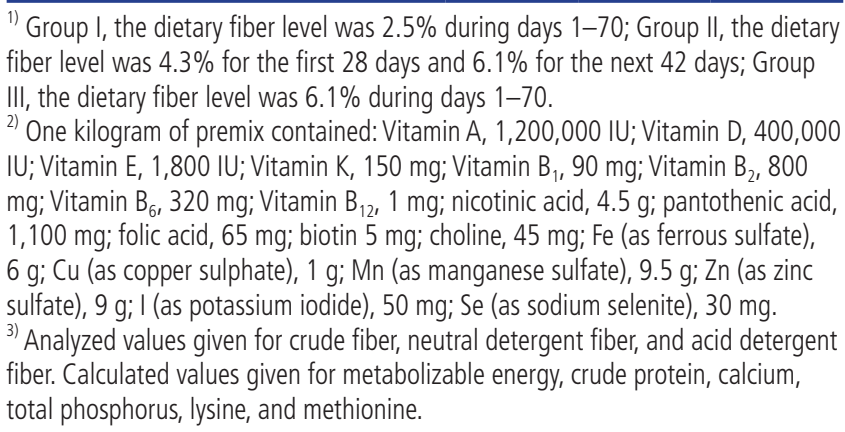 } \\
\hline
\end{tabular}

The Yangzhou University Animal Care and Use Committee approved all bird-handing protocols used in the study. The experiment was conducted using 468 healthy male Yangzhou goslings. The Yangzhou goose is a major species in China, which has medium body size, stable genetic performance, high reproductive rate, rapid early growth, good meat quality, strong stress tolerance, and amenability to coarse feed [8].

A total of 468 1-day-old healthy male Yangzhou goslings with similar body weight were randomly divided into 3 groups with 6 replicates per group and 26 geese per replicate. The geese in Groups I and III received diets with $2.5 \%$ and $6.1 \%$ dietary fiber during days 1-70, respectively. The geese in Group II received a diet with $4.3 \%$ dietary fiber for the first 28 days and $6.1 \%$ dietary fiber for the next 42 days. Water and feed were provided ad libitum. The geese were reared in the indoor house under similar environmental conditions (temperature: $24^{\circ} \mathrm{C} \pm 3^{\circ} \mathrm{C}$; relative humidity: $55 \% \pm 5 \%$; density: $0.5 \mathrm{~m}^{2} /$ goose), and all geese were exposed to natural daylight.

The composition and nutrient levels of the experimental diets are shown in Tables 1 and 2. The experimental diets were

Table 2. Ingredients and nutrient composition of experimental diets for days 29 to 70 (as fed)

\begin{tabular}{lccc}
\hline Item & Group I) $^{1)}$ & Group II) & Group III \\
\hline Ingredients (\%) & & & \\
Corn & 63.00 & 63.00 & 63.00 \\
Soybean meal & 25.30 & 25.30 & 25.30 \\
Cellulose & - & 3.76 & 3.76 \\
Limestone & 1.00 & 1.00 & 1.00 \\
Calcium hydrogen phosphate & 1.00 & 1.00 & 1.00 \\
Methionine & 0.16 & 0.16 & 0.16 \\
Salt & 0.30 & 0.30 & 0.30 \\
Vermiculite & 8.24 & 4.48 & 4.48 \\
Vitamin and trace mineral premix & 1.00 & 1.00 & 1.00 \\
Nutrient composition & & & \\
Metabolizable energy (MJ/kg) & 11.02 & 11.02 & 11.02 \\
Crude protein (\%) & 16.71 & 16.71 & 16.71 \\
Crude fiber (\%) & 2.50 & 6.15 & 6.15 \\
Neutral detergent fiber (\%) & 8.42 & 12.07 & 11.90 \\
Acid detergent fiber (\% & 3.13 & 6.78 & 6.67 \\
Calcium (\%) & 0.75 & 0.75 & 0.75 \\
Total phosphorus (\%) & 0.56 & 0.56 & 0.56 \\
Lysine (\%) & 0.82 & 0.82 & 0.82 \\
Methionine (\%) & 0.40 & 0.40 & 0.40 \\
\hline
\end{tabular}

1) Group I, the dietary fiber level was $2.5 \%$ during days 1-70; Group II, the dietary fiber level was $4.3 \%$ for the first 28 days and $6.1 \%$ for the next 42 days; Group III, the dietary fiber level was $6.1 \%$ during days $1-70$.

${ }^{2)}$ One kilogram of premix contained: Vitamin A, 1,200,000 IU; Vitamin D, 400,000 IU; Vitamin E, 1,800 IU; Vitamin K, 150 mg; Vitamin $B_{1}, 60$ mg; Vitamin $B_{2}, 600$ mg; Vitamin $\mathrm{B}_{6}, 200$ mg; Vitamin $\mathrm{B}_{12}, 1 \mathrm{mg}$; nicotinic acid, 3 g; pantothenic acid, $900 \mathrm{mg}$; folic acid, 50 mg; biotin, 4 mg; choline, $35 \mathrm{mg}$; Fe (as ferrous sulfate), $6 \mathrm{~g}$; Cu (as copper sulphate), $1 \mathrm{~g}$; Mn (as manganese sulfate), $9.5 \mathrm{~g}$; Zn (as zinc sulfate), $9 \mathrm{~g}$; I (as potassium iodide), $50 \mathrm{mg}$; Se (as sodium selenite), $30 \mathrm{mg}$.

${ }^{3)}$ Analyzed values given for crude fiber, neutral detergent fiber, and acid detergent fiber. Calculated values given for metabolizable energy, crude protein, calcium, total phosphorus, lysine, and methionine. 
formulated mainly according to the NRC [9] and prior research results from our laboratory $[10,11]$.

\section{Sample collection}

Body weight and feed intake by replicate were measured weekly from day 1 to $\mathrm{d} 70$. Average daily feed intake, average daily gain, and feed conversation rate were calculated at the end of the experiment. At day 70, two geese in each replicate with the average body weight of the replicate were selected. The selected geese were exsanguinated by a severing of the jugular vein and carotid artery on one side of the neck. The cecum was removed aseptically, clamped with forceps, and placed into sterile plastic bags on ice. After the cecum was opened longitudinally, cecal contents were immediately sampled and stored at $-70^{\circ} \mathrm{C}[12]$.

\section{DNA extraction}

After the frozen samples were thawed, the thawed samples that from the same treatment were fully mixed immediately. Genomic DNA was extracted from the mixed samples using a QIAamp DNA Stool Mini Kit (Tiangen Biotech Company Limited, Beijing, China) following the manufacturer's instructions. The DNA with an A260/280 ratio ranging between 1.8 and 2.0 was subjected to further analysis. DNA extracts were stored at $-70^{\circ} \mathrm{C}$.

\section{Polymerase chain reaction procedures}

$16 \mathrm{~S}$ rRNA genes were amplified by polymerase chain reaction (PCR) from the genomic DNA samples using specific primers for V3-V4 region of bacterial $16 \mathrm{~S}$ rRNA. The primer sequences were 341F (5'-CCTACGGGNGGCWGC AG-3') and 805R (5'-GACTACHVGGGTATCT AATCC-3') [13].

The PCR amplification reaction system (Tiangen Biotech Company Limited, Beijing, China) was as follows: $5 \mu \mathrm{L}$ of $10 \times$ PCR buffer, $0.5 \mu \mathrm{L}$ of dNTP, $10 \mathrm{ng}$ of genomic DNA, 0.5 $\mu \mathrm{L}$ of primer $\mathrm{F}, 0.5 \mu \mathrm{L}$ of primer $\mathrm{R}, 0.5 \mu \mathrm{L}$ of plantium Taq $(5$ $\mathrm{U} / \mu \mathrm{L}), \mathrm{ddH}_{2} \mathrm{O}$ was added to $50 \mu \mathrm{L}$. The PCR amplification reaction procedure was as follows: initial denaturation at $94^{\circ} \mathrm{C}$ for $3 \mathrm{~min}$; denaturation $94^{\circ} \mathrm{C}$ for $30 \mathrm{~s}$, annealing at $45^{\circ} \mathrm{C}$ for $20 \mathrm{~s}$, extension at $65^{\circ} \mathrm{C}$ for $30 \mathrm{~s}$, a total of 5 cycles; denaturation at $94^{\circ} \mathrm{C}$ for $20 \mathrm{~s}$, annealing at $55^{\circ} \mathrm{C}$ for $20 \mathrm{~s}$, extension at $72^{\circ} \mathrm{C}$ for $30 \mathrm{~s}$, a total of 20 cycles; extension at $72^{\circ} \mathrm{C}$ for 5 $\mathrm{min}$. Sequencing platform adopted in this study was Illumina MiSeq 2000 (Sangon Biotech Company Limited, Shanghai, China).

\section{Statistical analysis}

In this study, operational taxonomic unit (OTU) cluster analysis was used to classify the OTU sequences based on a 97\% similarity criterion. The community composition of each sample was shown at two different levels of phylum and genus, and bar charts were plotted using Excel. The abundance and diversity of microbiota were compared between each sample by calculating Chaol index, Shannon index and Simpson index.

\section{RESULTS}

\section{Performance}

The geese in Groups II and III had higher body weight at day 70 , higher average daily gain during day 1 to 70 , lower feed conversation rate during day 1 to 70 compared with those in Group I $(\mathrm{p}<0.05)$ [7].

\section{Alpha diversity index analysis}

The Alpha diversity index analysis of cecal microbiota in geese at day 70 is shown in Table 3. A total of 71,501 sequences and 4,913 OTUs were obtained by sequencing, with 23,524 sequences and 1,458 OTUs in Group I, 22,338 sequences and 1,581 OTUs in Group II, 25,639 sequences and 1,874 OTUs in Group III. In addition, Chaol index and Shannon index were decreased, whereas Simpson index of cecal microbiota of geese was increased in Group I compared with those in Groups II and III.

Microbial population analysis at the phylum level Microbial population analysis at the phylum level for cecal microbiota in geese at day 70 is shown in Figure 1. Relative abundance of Bacteroidetes and Firmicutes were decreased by $3.80 \%$ and $11.4 \%$, whereas relative abundance of Proteobacteria in the cecum of geese in Group I was increased by $64.3 \%$ compared with those in Group II. Relative abundance of Bacteroidetes and Firmicutes were decreased by $1.66 \%$ and $15.5 \%$, whereas relative abundance of Proteobacteria in the cecum of geese in Group I was increased by $60.6 \%$ compared with those in Group III.

\section{Microbial population analysis at the genus level}

Microbial population analysis at the genus level for cecal microbiota in geese at day 70 is shown in Figure 2. Relative abundance of Bacteroides and Parabacterioides decreased by $12.9 \%$ and $34.3 \%$, whereas relative abundance of Desulfovibrio in the cecum of geese in Group I increased by $24.6 \%$ compared with those in Group II. Relative abundance of Bacteroides and

Table 3. Diversity index of cecum microbiota in geese at day 70

\begin{tabular}{lccc}
\hline Item & Group I) $^{1)}$ & Group II) $^{\text {1) }}$ & Group III $^{1)}$ \\
\hline Total sequences & 23,524 & 22,338 & 25,639 \\
Total OUT & 1,458 & 1,581 & 1,874 \\
Chao1 & 8,995 & 11,557 & 9,221 \\
Shannon & 4.36 & 4.39 & 4.70 \\
Simpson & 0.041 & 0.038 & 0.031 \\
\hline
\end{tabular}

OTU, operational taxonomic unit.

1) Group I, the dietary fiber level was $2.5 \%$ during days 1-70; Group II, the dietary fiber level was $4.3 \%$ for the first 28 days and $6.1 \%$ for the next 42 days; Group III, the dietary fiber level was $6.1 \%$ during days $1-70$. 


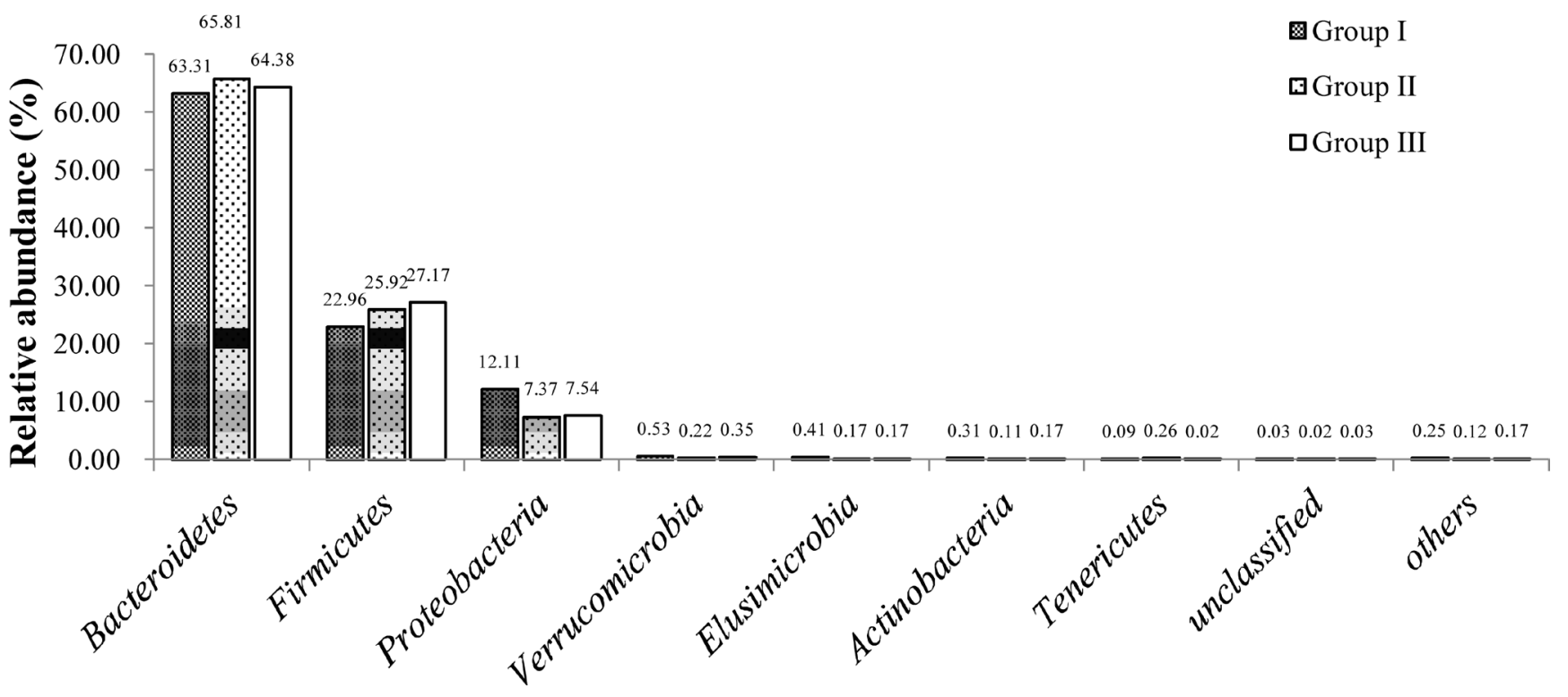

Figure 1. Relative abundance of cecal microbiota at the phylum level for geese at day 70 . Group I, the dietary fiber level was 2.5\% during days 1-70; Group II, the dietary fiber level was $4.3 \%$ for the first 28 days and $6.1 \%$ for the next 42 days; Group III, the dietary fiber level was $6.1 \%$ during days 1-70.

Parabacterioides decreased by $11.6 \%$ and $21.9 \%$, whereas relative abundance of Desulfovibrio in the cecum of geese in Group I increased by $42.0 \%$ compared with those in Group III.

\section{Similarity cluster analysis}

Similarity cluster analysis of cecal microbiota in geese at day 70 is shown in Figure 3. The distances between Group I and Group II, between Group I and Group III were greater than that between Group II and Group III.

\section{DISCUSSION}

Many studies have revealed that the gut microbiota can provide the host with short chain fatty acids, vitamin $\mathrm{E}$, and other benefits [14]. Furthermore, gut microbiota is useful for the development of the host immune system [15]. In the present study, we used high-throughput sequencing of the V3-V4 region of the $16 \mathrm{~S}$ rRNA gene to investigate the effects of dietary fiber levels on cecal microbiota composition in geese. The molecular analysis of $16 \mathrm{~S}$ rRNA genes has showed greater

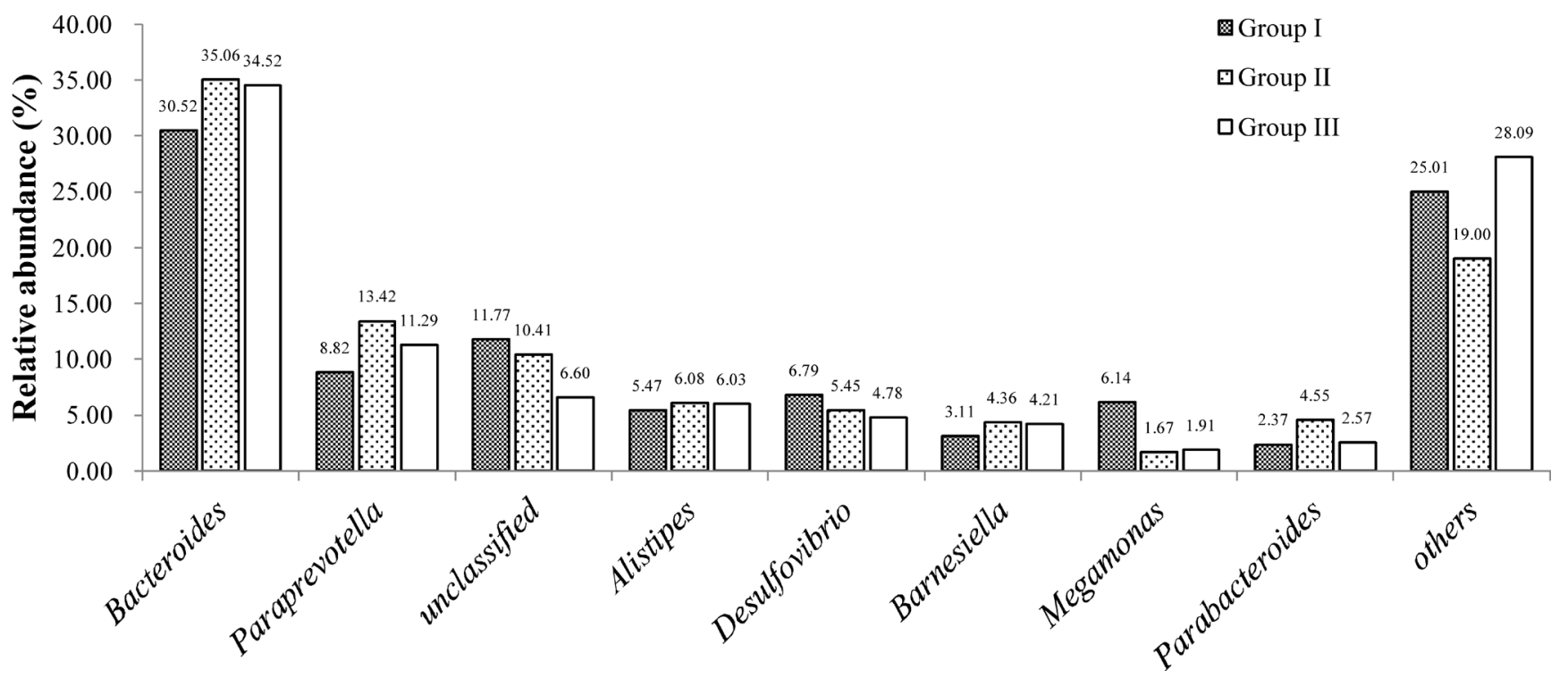

Figure 2. Relative abundance of cecal microbiota at the genus level for geese at day 70. Group I, the dietary fiber level was 2.5\% during days 1-70; Group II, the dietary fiber level was $4.3 \%$ for the first 28 days and $6.1 \%$ for the next 42 days; Group III, the dietary fiber level was $6.1 \%$ during days $1-70$. 


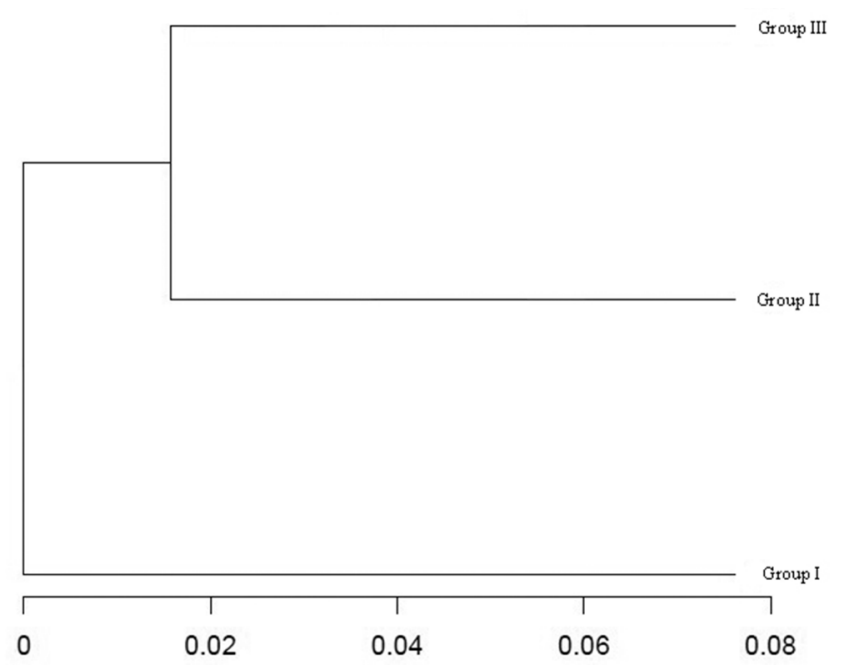

Figure 3. Similarity cluster analysis of cecal microbiota in geese at day 70 . Group I, the dietary fiber level was $2.5 \%$ during days 1-70; Group II, the dietary fiber level was $4.3 \%$ for the first 28 days and $6.1 \%$ for the next 42 days; Group III, the dietary fiber level was $6.1 \%$ during day $1-70$.

diversity of the microbial population than that achieved by culture-dependent methods [16]. The composition of cecal microbiota of animals can be significantly affected by diets [17].

The OTU data reflects the information of microbial species number. Shannon index and Simpson index reflect the microbial diversity in the sample $[18,19]$. The greater the Shannon value and the lower the Simpson index value means the higher microbial diversity [20]. In general, a more diverse microbial community shows stronger homeostasis of the intestinal microbial community and resistance to pathogens [21]. In addition, Chaol index reflects the abundance of microbiota [22]. In present study, low fiber level diet decreased the total OTUs, Shannon index, Chaol index, and increased Simpson index of cecal microbiota in geese at day 70 , indicating that low fiber level diet decreased numbers of microbial species, diversity of microbiota, and abundance of microbiota in cecum.

In present study, Bacteroidetes, Firmicutes, and Proteobacteria were the main bacteria in cecum of geese, which was consistent with the results of Qin et al [23]. Cellulolytic bacteria is not only rich in Firmicutes [24] but also rich in Bacteroidetes [25]. In addition, information on the microbial population at the genus level could further indicate the function of microbiota in intestinal tract. Paraprevotella has the ability to break down the polypeptide, starch, xylan and pectin into short chain fatty acids [26]. Short chain fatty acid has an important impact on intestinal health, and can protect the intestinal epithelium and inhibit the occurrence of enteritis and intestinal cancer [27]. In addition, Bacteroides plays an important role in helping decompose polyose and raise the utilization rate [28] to speed up development of the intestinal mucosa [29] and immune system, then raises the immunity of the host and maintains the balance of intestinal microecology [30]. In pre- sent study, low fiber level diet decreased the relative abundance of Firmicutes, Bacteroidetes, Bacteroides, and Paraprevotella in cecum of geese. In previous study, we observed that geese fed with low fiber diet had lower performance [7]. This inferred that the low fiber level diet decreased performance of geese might by reducing the relative abundance of beneficial microbiota in cecum.

In the graph of similarity cluster analysis, similarity was transformed into distance, to be convenient for observation. In present study, distances between Group I and Group II, between Group I and Group III was greater than that between Group II and Group III, indicating that the similarity of cecal microbiota between Group I and Group II, between Group I and Group III were smaller than that between Group II and Group III. That was consistent with the results of previous study that performance of geese fed with low fiber level diet was decreased compared with other groups. This also indicated that low fiber level diet affects performance by altering the diversity and population of cecal microbiota in geese.

\section{CONCLUSION}

The low fiber level (2.5\%) diet decreased diversity of microbiota, and relative abundance of some beneficial microbiota in cecum of geese at day 70 . The low fiber level diet might have negative influence on performance by altering the diversity and population of cecal microbiota in geese.

\section{CONFLICT OF INTEREST}

We certify that there is no conflict of interest with any financial organization regarding the material discussed in the manuscript.

\section{ACKNOWLEDGMENTS}

This work was financially supported by the China Agriculture Research System (CARS-42).

\section{REFERENCES}

1. Liu BY, Wang ZY, Wang HR, et al. Molecular profiling of bacterial species in the geese cecum. Czech J Anim Sci 2011;56: 192-203.

2. Mcnab JM. The avian caeca: a review. Worlds Poult Sci J 1973; 29:251-63.

3. Garcia DM. The role of the giant Canada goose (Branta Canadensis maxima) cecum in nutrition [master's thesis]. Columbia, MO, USA: University of Missouri-Columbia; 2006.

4. Yang HM, Wang ZY, Wang J, Shi SR, Zhu XH. Effects of caecectomy on digestibility of crude protein, calcium, phosphorus, neutral detergent fibre and acid detergent fibre in geese. Arch 
Geflügelkd 2009;73:189-92.

5. Chiou PW, Luz TW, Hsu C, Yu B. Effect of different sources of fiber on the intestinal morphology of domestic geese. AsianAustralas J Anim Sci 1996;9:539-50.

6. Hsu JC, Chen LI, Yu B. Effects of levels of crude fibre on growth performances and intestinal carbohydrases of domestic goslings. Asian-Australas J Anim Sci 2000;13:1450-4.

7. Li YP, Wang ZY, Yang HM, et al. Effects of dietary fiber on growth performance, slaughter performance, serum biochemical parameters, and nutrient utilization in geese. Poult Sci 2017; 96:1250-6.

8. Liu BY, Wang ZY, Yang HM, et al. Influence of rearing system on growth performance, carcass traits, and meat quality of Yangzhou geese. Poult Sci 2011;90:653-9.

9. NRC. Nutrient requirements of poultry. 9th rev. ed. Washington, DC, USA: National Academy Press; 1994.

10. Lu J, Kong XL, Wang ZY, et al. Influence of whole corn feeding on the performance, digestive tract development, and nutrient retention of geese. Poult Sci 2011;90:587-94.

11. Wang ZY, Yang HM, Lu J, Li WZ, Zou JM. Influence of whole hulled rice and rice husk feeding on the performance, carcass yield and digestive tract development of geese. Anim Feed Sci Technol 2014;194:99-105.

12. Apajalahti JH, Sarkilahti LK, Maki BR, et al. Effective recovery of bacterial DNA and percent-guanine-pluscytosine-based analysis of community structure in the gastrointestinal tract of broiler chickens. Appl Environ Microbiol 1998;64:4084-8.

13. Yang DH, Zhang YY, Du PC, et al. Rapid identification of bacterial species associated with bronchiectasis via metagenomic approach. Biomed Environ Sci 2014;27:898-901.

14. Stanley D, Hughes RJ, Moore RJ. Microbiota of the chicken gastrointestinal tract: influence on health, productivity and disease. Appl Microbiol Biotechnol 2014;98:4301-10.

15. Gensollen T, Iyer SS, Kasper DL, Blumberg RS. How colonization by microbiota in early life shapes the immune system. Science 2016;352:539-44.

16. Gong JH, Forster RJ, Yu H, et al. Molecular analysis of bacterial populations in the ileum of broiler chickens and comparison with bacteria in the cecum. FEMS Microbiol Ecol 2002;41:1719.

17. Rehman H, Hellweg P, Taras D, Zentek J. Effects of dietary inulin on the intestinal short chain fatty acids and microbial ecology in broiler chickens as revealed by denaturing gradient gel electrophoresis. Poult Sci 2008;87:783-9.

18. Shannon CE. The mathematical theory of communication. MD Computing: computers in medical practice 1963;14:306-17.

19. Simpson EH. Measurement of diversity. Nature 1949;163:688.

20. Wang Y, Sheng HF, He Y, et al. Comparison of the levels of bacterial diversity in freshwater, intertidal wetland, and marine sediments by using millions of illumina tags. Appl Environ Microbiol 2012;78:8264-71.

21. Konstantinov SR, Favier CF, Zhu WY, et al. Microbial diversity studies of the porcine gastrointestinal ecosystem during weaning transition. Anim Res 2004;53:317-24.

22. Chao A. Nonparametric estimation of the number of classes in a population. Scand J Statist 1984;11:265-70.

23. Qin J, Li R, Raes J, et al. A human gut microbial gene catalogue established by metagenomic sequencing. Nature 2010;464:5965.

24. Daly K, Stewart CS, Flint HJ, Shirazi-Beechey SP. Bacterial diversity within the equine large intestine as revealed by molecular analysis of cloned 16S rRNA genes. FEMS Microbiol Ecol 2001;38:141-51.

25. Matsui H, Kato Y, Chikaraishi T, et al. Microbial diversity in ostrich ceca as revealed by $16 \mathrm{~S}$ ribosomal RNA gene clone library and detection of novel Fibrobacter species. Anaerobe 2010;16:83-93.

26. Scheppach W, Luehrs H, Menzel T. Beneficial health effects of low-digestible carbohydrate consumption. Br J Nutr 2001; 85:S23-30.

27. von Rosenvinge EC, Song Y, White JR, et al. Immune status, antibiotic medication and $\mathrm{pH}$ are associated with changes in the stomach fliud microbiota. ISME J 2013;7:1354-66.

28. Backhed F, Ding H, Wang T, et al. The gut microbiota as an environmental factor that regulates fat storage. Am J Clin Nutr 2004;101:15718-23.

29. Stappenbeck TS, Hooper LV, Gordon JI. Developmental regulation of intestinal angiogenesis by indigenous microbes via paneth cells. Proc Natl Acad Sci USA. 2002;99:15451-5.

30. Sears CL. A dynamic partnership: celebrating our gut flora. Anaerobe 2005;11:247-51. 